\title{
Postoperative outcomes following surgery for deep endometriosis of the sacral plexus and sciatic nerve: a 52-patient consecutive series
}

\author{
Horace Roman ${ }^{1}$, Lise Dehan ${ }^{2}$, Benjamin Merlot ${ }^{1}$, Benoit Berby ${ }^{2}$, Damien Forestier ${ }^{1}$, Mikkel \\ Seyer-Hansen $^{3}$, Carole Abo ${ }^{2}$, and Jean-Jacques Tuech ${ }^{4}$ \\ ${ }^{1}$ Clinique Tivoli-Ducos \\ ${ }^{2}$ Centre Hospitalier Universitaire de Rouen \\ ${ }^{3}$ Aarhus Universitet \\ ${ }^{4}$ University Hospital, Rouen
}

July 21, 2020

\begin{abstract}
Background: Surgical management of deep endometriosis infiltrating pelvic nerves may allow an overall improvement in pain and neurologic disorders. Objective: To assess 1-year postoperative outcomes of surgery for deep endometriosis involving sacral roots and the sciatic nerve. Study Design: Retrospective study including 52 women undergoing surgery for deep endometriosis involving sacral roots and the sciatic nerve. We assessed 1-year postoperative outcomes. Results: Deep endometriosis involved sacral roots in 49 women $(94.2 \%)$ and the sciatic nerve in 3 cases (5.8\%). Sciatic pain was recorded in 43 women (82.7\%), pudendal neuralgia in 11 women $(21.2 \%)$ and leg motor weakness in 14 cases $(27 \%)$. Surgical procedures carried out on pelvic nerves included complete releasing and decompression (92.3\%), excision of epineurium (5.8\%) and intraneural excision (1.9\%). Additional procedures involved the digestive tract in $82.7 \%$ of cases and the urinary tract in $46.2 \%$. Rectovaginal fistula occurred in $13.5 \%$ of cases. Self-catheterisation was required in 14 cases (27\%) at 3 weeks after surgery, and in only 3 women (5.8\%) 12 months later. One-year follow up showed significant improvement in quality of life using SF36 and standardised gastrointestinal scores. De novo hypoesthesia, hyperaesthesia or allodynia were recorded in 9 women (17.2\%). The cumulative pregnancy rate was $77.2 \%$ following natural conception in $47 \%$. Conclusions: Laparoscopic management of deep endometriosis involving sacral roots and the sciatic nerve improves patient symptoms and overall quality of life. Although pain reduction may be rapid following surgery, other sensory or motor complaints including bladder dysfunction may be recorded over months or years.
\end{abstract}

\section{Introduction}

Deep rectovaginal endometriosis nodules in young women may involve pelvic nerves, particularly when there is infiltration of the parametrium, with or without adjacent vagina, rectum or ureters. Parametrial nodules may compress, surround or infiltrate nerves of large diameter, such as sacral roots, sciatic or pudendal nerves, or thin fibres such as splanchnic or hypogastric nerves and the inferior or superior hypogastric plexus. Depending on the role of nerve fibres, somatic and vegetative symptoms may occur, such as pain in buttocks, legs or perineum, skin hypo or hyperesthesia, bladder and rectal dysfunction or vaginal dryness. These relatively rare symptoms may be overlooked by gynaecologists or considered unrelated to nerve involvement in a severe endometriosis ${ }^{1}$.

Over the past two decades, several authors have reported series of patients surgically managed for endometriosis with various degrees of involvement of sacral roots and the sciatic nerve ${ }^{1-3}$. Large diameter nerve involvement generally occurs in two main ways, which in some patients may be associated. i) The first and most frequent situation concerns large rectovaginal or uterosacral nodules, which develop laterally 
through the parametrium to the lateral pelvic wall, pyriformis and levator ani muscles, where they come into contact with the sacral plexus (Fig 1a). These nodules involve not only the sacral roots, but also the inferior hypogastric plexus, splanchnic and hypogastric nerves, as well as low rectum, vagina or homolateral ureter. Patients presenting with this type of nodule usually present bladder, rectum and left colon dysfunction in addition to vaginal dryness, more likely due to the involvement of the inferior hypogastric plexus and thin nerves than to that of sacral roots. They may also report somatic pain, particularly in the buttock, perineum and leg, due to involvement of sacral root S2, S3 and S4, which send fibres into the sciatic and pudendal nerves; ii) the second less frequent situation involves deep endometriosis nodules which occur more laterally and cranially, in contact with the pelvic wall and sciatic nerve, before exit through the greater sciatic foramen (Fig 1b). Patients present somatic, sensory (buttock and leg pain) and motor complaints (alteration of the Achillean reflex and foot drop, due to involvement of S1). Conversely, they usually have no vegetative symptoms, due to no involvement of sympathetic hypogastric nerves and parasympathetic fibres originating from S2-S4, and no associated infiltration of rectum, ureters and vagina ${ }^{4}$.

Outcomes of surgical management of deep endometriosis infiltrating the large nerves have been reported by several authors in the literature, and considered satisfactory in the majority of $\operatorname{cases}^{1,2,4}$. To further knowledge in this field, our study aimed to assess preoperative complaints, intraoperative findings, surgical procedures and postoperative outcomes in a series of consecutive patients managed for deep endometriosis involving sacral roots and the sciatic nerve.

\section{Patients and methods}

We enrolled in this series all patients with deep endometriosis involving sacral roots and the sciatic nerve, who had benefited from surgical management carried out over 40 consecutive months from October 2016 to April 2019 at Rouen University Hospital, the Endometriosis Centre, Clinique Tivoli-Ducos, Bordeaux, France and Aarhus University Hospital, Denmark. Inclusion criteria were: i) deep endometriosis nodule with limits that come into contact with, entrap or infiltrate sacral roots or the sciatic nerve shown on pelvic MRI, in axial, coronal and sagittal views; ii) presence of vegetative (bladder, rectal dysfunction) or somatic complaints (pain in the leg, buttock or perineum; motor weakness) attributable to pelvic nerve involvement; iii) surgical procedure requiring complete dissection, shaving or intraneural excision of endometriosis of sacral roots or sciatic nerve; iv) patient inclusion and follow up in CIRENDO database. Exclusion criteria were: i) non-French nationality and non-English speaking patients, ii) patients not fulfilling at least one inclusion criteria.

All patients were preoperatively examined by a gynaecologist surgeon whose practice exclusively focuses on deep endometriosis (H.R). Preoperative assessment was performed by radiologists with considerable experience in deep endometriosis and included pelvic MRI, along with endorectal/transvaginal ultrasound, computed tomography based virtual colonoscopy, uro-CT or uro-MRI when required. Urodynamic assessment was performed in patients with baseline symptoms suggesting significant bladder dysfunction. Surgery was proposed in patients for whom symptoms were not relieved by medical therapy. Principles of surgical approach were preoperatively discussed, and patients were fully informed about the aims, risks and expected benefits of the surgery.

All patients were managed laparoscopically. The approach for deep endometriosis infiltrating the parametrium down to the sacral plexus is standardised (Supplemental Video 1) and follows successive steps: 1) ureterolysis; 2) opening of the pararectal space on contact with the rectum in a sagittal plane; 3) dissection prolonged toward the rectovaginal space, including the section of rectovaginal nodules in one fragment infiltrating the rectum, and another infiltrating the vagina and the parametrium, down to levator ani muscles; 4) dissection of presacral space, identification of the superior hypogastric plexus and hypogastric nerve; 5) incision of the peritoneum at promotorium level, prolonged laterally above the origin of hypogastric vessels; 6) anterograde dissection of hypogastric artery and identification of hypogastric vein; 7) anterograde dissection of hypogastric vein and opening of Okabayashi space, successive identification and when required, ligation of pelvic veins reaching the hypogastric vein. This step is of major importance because the surgeon should 
be able, at any moment, to ligate the hypogastric vein in case of dissection-related injury; 8) dissection is prolonged behind venous network, with identification of pyriform muscles and sacral root S2, S3 and S4, as well as that of splanchnic nerves originating from sacral roots and reaching the inferior hypogastric plexus; 9) anterograde dissection of the nerve network and inferior hypogastric plexus, up to posterior limits of the deep endometriosis nodule; 10) excision of deep endometriosis nodule, from the posterior limit, to the inferior limit on contact with sacral roots, which should be released or shaved, then to the lateral limit on contact with pyriform muscle and lateral pelvic wall. During this step, dissection is less standardised, and follows nodule limits step by step, with sacrifice of involved fibres depending on inferior hypogastric plexus and ligation of several parametrial veins; 11) excision of adjacent vaginal infiltration; 12) removal of rectal infiltration using shaving, disc excision or segmental resection.

Management of deep endometriosis nodules infiltrating the sciatic nerve employs a different approach, laterally from external iliac vessels (5). The procedure includes the following steps (Supplemental Video 2): 1) longitudinal incision of the peritoneum covering external iliac artery; 2) opening and dissection of the space identified laterally by the psoas muscle and medially by the external iliac artery and vein; 3) the dissection progresses more deeply, with identification of the obturatory nerve which is pushed medially, on contact with the psoas muscle; 4) opening of the lumbosacral space, below the level of obturatory nerve, and identification of the sciatic nerve, resulting from the confluence of L5, S1 and S2 roots. During this step the deep endometriosis nodule is identified; 5) dissection of the nodule, in lateral direction on contact with the muscle, up to the greater sciatic notch; 6) removal of the nodule by progressive dissection on contact with sciatic nerve; haemostasis of surrounding gluteal veins should employ clips as coagulation may be imperfect and veins may retract after section, resulting in hidden bleeding which may be challenging to control; 7) complete removal of nodule allows exposure of pudendal nerve, located medially and caudally, just before its exit through the lesser sciatic foramen.

Associated localisations of the disease were managed during the same procedure. We performed shaving, disc excision or segmental resection to remove colorectal nodules, depending on nodule features ${ }^{6}$. Deep endometriosis responsible for stenosis of ureters was managed by ureterolysis or ureteral resection, followed by either end-to-end ureteral anastomosis or reimplantation of the ureter into the bladder, depending on the extrinsic or intrinsic infiltration of the ureter. Ovarian endometriomas were treated by vaporisation using plasma energy, cystectomy, sclerotherapy or drainage, depending on ovarian reserve and further intention to conceive. Other endometriosis localisations were routinely removed by excision, until all macroscopic lesions were treated. Following the procedure, the surgeon completed a surgical questionnaire with responses recorded in the CIRENDO database. An item "sacral plexus involvement" allowing recording of patients managed with this localisation was added in January 2016.

Postoperative hospitalisation varied from 4 to 6 days. Clinical symptoms and body temperature were recorded 3 times/day, and assessment of blood values of C-reactive protein (CRP) and white blood cells (WBC) was routinely performed at day 4, 5 and 6 . Administration of pregabalin by progressive doses began day 1 after surgery and stopped after two weeks, only if neuropathic pain, paraesthesia or hypo/hyperaesthesia were completely absent. The urinary catheter was removed on postoperative days 1 or 2 and post-voiding bladder residue (PVR) was recorded using an ultrasonic bladder scanner (BladderScan BVI 6100, Verathon Inc, Bothell, WA, USA) after each spontaneous void or every 4 hours. Any PVR greater than $100 \mathrm{~mL}$ was considered abnormal and further bladder scanning was performed. If PVR was greater than $400 \mathrm{~mL}$, the bladder was emptied using an 'in out' intermittent catheter. If the patient was completely unable to urinate, routine intermittent catheterisation was carried out 5 times per day (at $7 \mathrm{am}, 11 \mathrm{am}, 2: 30 \mathrm{pm}$, $6 \mathrm{pm}$ and 10pm). When PVR remained high (over 100-150 ml) 3 to 4 days after surgery, patients were asked to perform intermittent self-catheterisation 5 times per day following discharge. Alfuzosine $10 \mathrm{mg} /$ day was prescribed for 3 months to reduce urethral opening pressures. One month postoperatively, patients were seen with a 48-hour record of urinary volumes voided spontaneously or drained via self-catheterisation. If PVR was less than $100 \mathrm{ml}$, self-catheterisation was ceased; if PVR was greater than $100 \mathrm{ml}$, self-catheterisation was continued for a month or more. 
PVRs during hospitalisation and during the period of self-catheterisation were carefully recorded in patient medical charts. Postoperative complications and the duration of self-catheterisation required were recorded in the CIRENDO database, as well as follow up data (pain, desire to conceive, pregnancies, standardised questionnaires on gastrointestinal and urinary outcomes and quality of life).

Prospective recording of data concerning antecedents, clinical symptoms, findings of clinical examinations and diagnostic imaging, surgical procedures and postoperative outcomes was performed through the CIRENDO (North-West Inter Regional Female Cohort for Patients with Endometriosis) database (NCT02294825). This prospective cohort is financed by the G4 Group (The University Hospitals of Rouen, Lille, Amiens and Caen) and coordinated by one of the authors (H.R.). Information was obtained using self-questionnaires and surgical and histological records, while data recording, contact and follow-up were carried out by 2 clinical research technicians. To ensure follow up, women enrolled in the database were asked to answer questionnaires 1, 3, 5, 7 and 10 years after surgery. Standardised gastrointestinal questionnaires were routinely used to assess pre- and post-operative digestive and urinary function: the Gastrointestinal Quality of Life Index (GIQLI) ${ }^{7}$, the Knowles-Eccersley-Scott-Symptom Questionnaire (KESS) ${ }^{8}$, the Wexner anal continence score ${ }^{9}$ and the Urinary Symptom Profile scale (questions $8^{\text {th }}, 9^{\text {th }}$ and $10^{\text {th }}$ exploring bladder dysfunction) ${ }^{10}$. Quality of life was assessed using the SF-36 score ${ }^{11}$. Prospective recording of data was approved by the French authority CCTIRS (Advisory Committee on information processing in healthcare research).

Statistical analysis was performed using Stata 11.0 software (StatCorp). Patient characteristics, surgical procedures, postoperative outcomes and score values were presented as numbers and percentages (qualitative variables) or mean and SD (continuous variables). Comparison between baseline and 1-year follow up data was performed using paired Student t test (continuous variables) and McNemar test with correcting for continuity (qualitative variables). The study was approved by the Rouen University Hospital Institutional Ethics Committee for Non-Interventional Research (E2020-21, April 13, 2020).

\section{Results}

From January 2016 to April 2019, 52 patients met inclusion criteria and were included in our retrospective study. Their data were prospectively recorded in the CIRENDO database. Forty-two patients were managed in Rouen from January 2016 to June 2018, 1 patient in Aarhus in October 2017 and 9 patients underwent surgery in Bordeaux, from September 2018 to April 2019.

Table 1 presents patient characteristics. $38.5 \%$ of patients had past history of surgery for endometriosis, using an open abdominal approach in a quarter of cases and including major procedures such as colorectal resections or nephrectomy. A majority of patients $(80.8 \%)$ were nullipara, $65.4 \%$ of women expressed pregnancy intention before surgery, and in 50\% infertility was affirmed. Symptoms presumed to be related to sacral roots or sciatic nerve involvement were various and included somatic pain, paraesthesia, motor weakness, bladder dysfunction and severe constipation. Twenty-seven patients $(51.9 \%)$ had at least one symptom suggesting bladder dysfunction (USP score $>=1$, at least "abdominal contraction required" OR "weak urine flow" OR "urination difficult to start or to finish"), in 13 patients (25\%) bladder voiding alteration was significant with USP score $>=3$ (at least "abdominal contraction required" AND "weak urine flow" AND "urination difficult to start or to finish"), while in 1 patient (1.9\%) urination had become impossible and bladder voiding was exclusively performed using self-catheterisation (USP $=9$ ).

Table 2 presents intraoperative findings, surgical procedures and postoperative complications. A majority of patients presented with large nodule of the parametrium (94.2\%), providing an explanation for the high rate of concomitant digestive tract infiltration, particularly of the rectum (84.6\%), the frequency of large vaginal infiltrations ( $80.8 \%$ of patients had vaginal nodules over $3 \mathrm{~cm}$ in width) and frequent lesions localised in the urinary tract (46.2\%). Surprisingly, ovaries and fallopian tubes were rarely involved. A third of patients had an AFSr score $<=15$, and only one third of patients underwent a procedure for ovarian endometrioma. Conversely, procedures on the digestive tract were carried out in $82.7 \%$ of patients, half of whom had a preventive stoma.

In 9 patients out of 10, sacral root and splanchnic nerve involvement could be removed by sacral root 
and sciatic nerve dissection, with large endometriosis nodule involvement leading to the sacrifice of inferior hypogastric plexus. In 3 cases, nerves were shaved using cold scissors and excision was performed inside the epineurium. In one patient, endometriosis appeared to infiltrate the interior of the nerve and complete excision led to a $5 \mathrm{~mm}$ wide hollow area inside the nerve. Postoperative outcomes were however uneventful.

Four intraoperative complications $(7.7 \%)$ were recorded, related to severe haemorrhage due to hypogastric vein injuries. In 2 cases, the vein was ligated laparoscopically. For the other 2 patients whose past history of colorectal resection for endometriosis resulted in postoperative extensive fibrosis, haemorrhage could not be managed laparoscopically and conversion to open surgery was required.

The high rate of rectovaginal fistula was related to a high frequency of excision of mid or low rectum $(80.8 \%)$ associated with colpectomy $(84.6 \%)$. Bladder dysfunction over 3 weeks was recorded in $27 \%$ of patients, however only $5.8 \%$ of them still required self-catheterisation 12 months after surgery and one of them benefited from neuromodulation of sacral roots. Conversely, 6 patients (11.5\%) presented with postoperative transitory urinary stress incontinence.

Table 3 presents data recorded 1 year postoperatively. An overall improvement in somatic pain and motor disorders was affirmed by 47 patients $(90.4 \%)$ and confirmed by the significant increase in SF-36 values assessed 1 year after surgery. An overall improvement in terms of digestive function was shown by better gastrointestinal score values. Conversely, there was a tendency towards an overall impairment of bladder function. While surgical procedures performed on nerves were followed by a tendency towards a reduction in the rate of patients experiencing paraesthesia and motor weakness in the legs, some patients presented new complaints such as hypoesthesia, hyperesthesia or allodynia.

Twenty-two patients (44.2\%) attempted to conceive during postoperative follow up of $23+/-8.7$ months. The cumulative pregnancy rate was $77.2 \%$ (17 patients) following natural conception in $47 \%$ (8 patients) and ART management in $53 \%$ (9 patients) of cases. Fourteen women delivered $(60.9 \%)$, and 3 pregnancies were ongoing at the time of manuscript submission (13\%). At the time of manuscript submission, two patients were benefiting from ongoing ART management (8.7\%) while 4 other patients had deferred pregnancy attempts following unsuccessful ART management (17.4\%).

\section{Discussion}

We report a series of patients with deep endometriosis involving the sacral plexus or sciatic nerve, a complex disease which in a majority of cases involves other major pelvic organs before reaching pelvic nerves. The resulting diverse clinical presentations, due to multiple deep endometriosis localisations, required complex surgical procedures carried out by multidisciplinary surgical teams, with these procedures related to a significant risk of postoperative complications. A year after surgery, despite an overall improvement in pain and quality of life and a high pregnancy rate by natural conception in half of the cases, some patients experienced persistent bladder dysfunction and neurological symptoms directly related to the surgical manipulation of nerves. Due to the relative rarity of sacral and sciatic nerve endometriosis, we believe that our data may provide additional helpful information to existing knowledge available in the literature.

Our series has several weaknesses. Firstly it is a retrospective series of patients receiving surgical management, without a control group. In our practice we previously treated two patients for whom pelvic MRI revealed deep endometriosis infiltrating pelvic wall and entrapping the sacral plexus, and two others with deep endometriosis of the lumbosacral space involving the sciatic nerve. All four patients received medical treatment based on continuous contraceptive pill intake resulting in prolonged amenorrhea, and consider their residual symptoms and quality of life as satisfactory. These patients are however not included in the CIRENDO database as they have not had surgery in our department. Furthermore their endometriosis localisations have not intraoperatively been confirmed and the patient number is too small to allow any comparison with the cohort of women who benefited from surgery. Due to the lack of control group, we cannot definitively state that deep endometriosis removal leads to a significant improvement in pain and quality of life when compared to continuous medical treatment, though all 52 women underwent surgery due to the lack of efficacy of previous medical therapies. 
The second limit is related to the heterogeneity of multiple deep endometriosis localisations recorded in our series and difficulties in demonstrating a precise relationship between specific localisations and corresponding symptoms. Accordingly baseline severe constipation may be attributed to mid/low rectum infiltration by deep endometriosis, or to splanchnic nerves or sacral root S2-S4 involvement; while postoperative constipation may result from postoperative low rectal resection syndrome (LARS) ${ }^{12}$ or rectal denervation following extensive removal of parametrium together with splanchnic nerves ${ }^{6}$. Consequently, assessment of the true efficiency of nerve surgical management on pelvic organ function may be challenging.

Our series presents however several strengths. This is a case-series including consecutive patients with strict inclusion criteria and prospective recording of preoperative, intraoperative and postoperative data, employing standardised questionnaires to assess pelvic pain, digestive and urinary function and quality of life. There is no loss to follow up. Inclusion criteria limited enrolment to only women for whom deep endometriosis nodules were in close contact with the sacral plexus or sciatic nerve, requiring specific nerve dissection or excision. Our results cannot therefore be overestimated by inclusion of women with neurological complaints associated with a less severe disease. All procedures were fully recorded and are available for review, and 5 procedures were broadcasted live during surgical meetings.

Our study provides detailed data on intraoperative findings and postoperative outcomes in patients managed for deep endometriosis involving large pelvic nerves. Due to the low prevalence of these localisations, clinical study available in the literature tends to rely on case-series, despite the afore-mentioned design weaknesses. The pooling of information reported by several surgical teams allows to further knowledge in this field and may be helpful for colleagues interested in the management of such patients.

Deep endometriosis infiltrating large pelvic nerves has been described as far back as $1955^{13}$, but it was 50 years later that the first description of a laparoscopic approach to release the sacral plexus and sciatic nerve with deep endometriosis involvement was reported ${ }^{5}$. Successive case-series confirmed overall pain improvement in patients having benefited from surgery. The first large case series including 27 women with isolated endometriosis of the sciatic nerve and 148 women with deeply infiltrating parametric endometriosis with sacral plexus infiltration, was published by Possover et $\mathrm{al}^{2}$, and recorded a decrease in mean VAS pain score from 7.7 to 2.6. Later, the Negrar Hospital team (Verona, Italy) published several technical reports, focusing on the anatomy of pelvic nerves and the nerve-sparing technique ${ }^{3,14}$. Lemos et al reported on their experience in the management of 13 women with deep endometriosis responsible for the entrapment of lumbosacral plexus, and noted an overall improvement in 6 patients, while 6 other women were completely pain-free ${ }^{1}$. More recently, Possover et al reported a series of 46 women managed for intraneural endometriosis of the sciatic nerve which required resection of more than $30 \%$ of the nerve. Follow up continued over 5 years and revealed significant pain reduction and recovery of nerve function ${ }^{4}$.

Deep endometriosis nodules involving sacral roots differ from those of the sciatic nerve. Firstly, there is a higher prevalence of sacral roots involvement representing $94.2 \%$ of cases in our series and 85\% (148 out of 175 cases) for Possover et $\mathrm{al}^{2}$. Secondly sacral roots entrapment appears to be much more frequent than actual nerve infiltration, due to the posterior development of large rectovaginal or parametrial nodules. Conversely, intraneural endometriosis was more frequently reported in sciatic nerves and may require partial resection of the nerve ${ }^{4}$.

Although pain reduction may occur early after surgery, various sensory or motor complaints may continue over months or years. When preoperatively affected, motor function recovery is long, and normal gait may take up to 5 years to restore ${ }^{4}$. Nerve entrapment or infiltration by fibrous endometriosis nodules require meticulous nerve dissection, excision of epineurium, fibre manipulation, as well as repeated haemostasis procedures responsible for thermal diffusion into the nerve, oedema and limited ischemia. These circumstances unavoidably lead to neuropraxia ${ }^{15}$ followed by postoperative sensory, motor or vegetative temporary disorders. Neuropraxia refers to mild nerve injury involving impairment of both motor and sensory functions and is the first type of peripheral nerve injury according to the Seddon and Sunderland classification ${ }^{16,17}$ with no loss of axon but a temporary loss of myelin sheath. This demyelination leads to impairment of impulse conduction across the nerve segment ${ }^{15}$ without Wallerian degeneration. The prognosis is favourable with 
complete recovery likely within weeks or months, due to axon remyelination ${ }^{18}$. Transitory bladder dysfunction requiring self-catheterisation for 4 to 6 weeks is a common clinical outcome of neuropraxia involving sacral or splanchnic nerves, and provides an explanation for bladder function recovery in three quarters of our patients with routine self-catheterisation during the early postoperative period. Administration of neuroleptic agents and postoperative physiotherapy play a key role in improving nerve recovery ${ }^{4}$.

However, neuropraxia is unlikely to be the most severe complication recorded in patients managed for deep endometriosis of the sacral plexus, particularly when the disease also involves low rectum, vagina or ureters. Concomitant rectal excision may be followed by specific complications such as rectovaginal fistula or low anterior rectal resection syndrome $(\mathrm{LARS})^{12}$. Although our rectovaginal fistula rate may be considered high, it remains comparable to that reported in other series of patients undergoing low rectal resection for deep endometriosis associated with colpectomy ${ }^{19}$. The high rate of vaginal involvement requiring colpectomy also led to the relatively high prevalence of early postoperative pelvic abscess and frequent use of preventive stoma.

Nerve-sparing surgery, commonly applied in oncology for preservation of autonomic nerves, has been shown to improve quality of life by reducing the incidence of functional complications without compromising long-term survival $^{20}$. The approach has to be tailored to suit surgical management of deep infiltrating endometriosis, which differs from cancer ${ }^{14,21}$. In cases where surgical indication exists for the treatment of early cancer, this approach works in disease-free tissue where nerves are not involved by the disease and may thus be easily separated and preserved. Conversely, preservation of the inferior hypogastric plexus is not possible and has to be sacrificed in patients who present with large infiltrations of the parametrium down to the levator ani muscles and sacral plexus. As performing small nerve sparing in large nodules of the parametrium is not feasible, bladder or rectal functional impairment may only be avoided by preserving the contralateral inferior hypogastric plexus, hypogastric and splanchnic nerves. In cases where endometriosis involves both parametria, surgeons tend to favour perfroming complete resection on the side with the more aggressive disease, and lesser treatment on the contralateral side, thereby preventing complete denervation of the bladder, rectum and vagina ${ }^{1,2,21}$. While unilateral sacrifice of small pelvic nerves may be considered, the conservation of large sacral and sciatic nerves remains a priority so as to avoid major sensory and motor somatic troubles.

It should be noted that women with deep endometriosis involving the sacral plexus and sciatic nerve in our series rarely had ovarian and fallopian tubes localisations, their disease being mainly sub-peritoneal. This resulted in an excellent pregnancy and delivery rate as early as 1 year after the procedure, with natural conception in half of the cases. This point is of particular importance, as a fear of fertility impairment following a complex surgical management may lead to unnecessarily privileging first line IVF in symptomatic patients with such severe disease and pregnancy intention.

All authors recommend referring patients with deep endometriosis of the sacral plexus and sciatic nerve to expert centres with high volume activity ${ }^{4,14,22}$. This recommendation highlights parameters which are important for ensuring the best outcomes: knowledge of anatomy and laparoscopic approaches to the sacral plexus, the availability of multidisciplinary teams for treatment of associated localisations in the digestive and urinary tract, experience in surgical procedures that ensure organ function preservation for each localisation, an ability to perform surgery in a reasonable operative time, and an ability to identify the early signs and symptoms of potential postoperative complications and to accurately manage them without delay. We believe this recommendation to be reasonable, despite the present lack of a large multicenter series or randomized trial to support it.

Study funding/competing interest(s): The North-West Inter Regional Female Cohort for Patients with Endometriosis (CIRENDO) is financed by the G4 Group (The University Hospitals of Rouen, Lille, Amiens and Caen) and ROUENDOMETRIOSE Association. No financial support was received for this study. The authors declare no competing interests related to this study.

\section{Authors' roles:}


Horace Roman and Lise Dehan designed the trial and wrote the first draft of the manuscript. Horace Roman, Benjamin Merlot, Mikkel Seyer-Hansen, Damien Forestier, and Jean-Jacques Tuech performed the surgery. Lise Dehan, Benoit Berby and Carole Abo performed statistical analysis and data collection. All the authors contributed to the final manuscript.

\section{Details of ethical approval:}

All patients signed an informed consent before enrollment in CIRENDO database and the study was approved by the Committé d'Ethique de la Recherche Non Interventionnelle, Rouen University Hospital (E2020-21, April 13, 2020).

\section{Tables and Figures:}

Table 1. Patient' characteristics and main baseline complaints.

Table 2. Intraoperative data and early postoperative outcomes.

Table 3. Postoperative outcomes 1 year after surgery.

Figure 1. MRI findings in deep endometriosis entrapping sacral roots (a) and the sciatic nerve (b) and corresponding intraoperative views.

Supplemental Video 1. Main steps of laparoscopic excision of deep endometriosis involving sacral roots.

Supplemental Video 2. Main steps of laparoscopic excision of deep endometriosis involving right sciatic nerve.

\section{References}

1. Lemos N, Souza C, Marques RM, Kamergorodsky G, Schor E, Girão MJ. Laparoscopic anatomy of the autonomic nerves of the pelvis and the concept of nerve-sparing surgery by direct visualization of autonomic nerve bundles. Fertil Steril. 2015;104:e11-2.

2. Possover M, Schneider T, Henle KP. Laparoscopic therapy for endometriosis and vascular entrapment of sacral plexus. Fertil Steril 2011; 95: 756-8.

3. Ceccaroni M, Clarizia R, Alboni C, Ruffo G, Bruni F, Roviglione G, Scioscia M, Peters I, De Placido G, Minelli L. Laparoscopic nerve-sparing transperitoneal approach for endometriosis infiltrating the pelvic wall and somatic nerves: anatomical considerations and surgical technique. Surg Radiol Anat. 2010;32:601-4.

4. Possover M. Five-year follow-up after laparoscopic large nerve resection for deep infiltrating sciatic nerve endometriosis. J Minim invasive Gynecol 2017; 24: 822-6.

5. Possover M, Baekelandt J, Flaskamp C, Li D et al. Laparoscopic neurolysis of the sacral plexus and the sciatic nerve for extensive endometriosis of the pelvic wall. Minim Invasive Neurosurg 2007; 50:33-36

6. Roman H, Bubenheim M, Huet E, Bridoux V, Zacharopoulou C, Daraï E, Collinet P, Tuech JJ. Conservative surgery versus colorectal resection in deep endometriosis infiltrating the rectum: a randomized trial. Hum Reprod. 2018;33:47-57.

7. Nieveen van Dijkum EJM, Terwee CB, Oosterveld P, van der Meulen JHP, Gouma DJ, de Haes JCJM. Validation of the gastrointestinal quality of life index for patients with potentially operable periampullary carcinoma. Br J Surg 2000; 87: 110-5.

8. Knowles CH, Eccersley AJ, Scott SM, Walker SM, Reeves B, Lunniss PJ. Linear discriminant analysis of symptoms in patients with chronic constipation. Validation of a new scoring system (KESS). Dis Colon Rectum 2000; 43: 1419-26.

9. Jorge JM, Wexner SD. Etiology and management of fecal incontinence. Dis Colon Rectum1993; 36: 77-97.

10. Haab F, Richard F, Amarenco G, et al. Comprehensive evaluation of bladder and urethral dysfunction symptoms: development and psychometric validation of the Urinary Symptom Profile (USP) questionnaire. Urology 2008;71:646-56.

11. Brazier JE, Harper R, Jones NMB, et al. Validating the SF-36 health survey questionnaire: new outcome measure for primary care. BMJ 1992;305:160-4. 
12. Emmertsen KJ, Laurberg S. Low anterior resection syndrome score: development and validation of a symptom-based scoring system for bowel dysfunction after low anterior resection for rectal cancer. Ann Surg 2012;255:922-8.

13. Denton RO, Sherrill JD. Sciatic syndrome due to endometriosis of sciatic nerve. South Med J. 1955;48:1027-31.

14. Ceccaroni M, Clarizia R, Roviglione G, Ruffo G. Neuro-anatomy of the posterior parametrium and surgical considerations for a nerve-sparing approach in radical pelvic surgery. Surg Endosc. 2013;27:438694.

15. Kamble N, Shukla D, Bhat D. Peripheral Nerve Injuries: Electrophysiology for the Neurosurgeon. Neurol India. 2019;67:1419-1422.

16. Seddon HJ. A classification of nerve injuries. Br Med J 1942;2:237-9.

17. Sunderland S. The anatomy and physiology of nerve injury. Muscle Nerve 1990;13:771-84.

18. Bernsen HJ, Koetsveld A, Frenken CW, van Norel GJ. Neuropraxia of the cervical spinal cord following cervical spinal cord trauma: A report of five patients. Acta Neurol Belg 2000;100:91-5

19. Belghiti J, Ballester M, Zilberman S, Thomin A, Zacharopoulou C, Bazot M, Thomassin-Naggara I, Darai E. Role of protective defunctioning stoma in colorectal resection for endometriosis. J Minim Invasive Gynecol. 2014;21:472-9.

20. Long Y, Yao DS, Pan XW, Ou TY et al., Clinical efficacy and safety of nerve-sparing radical hysterectomy for cervical cancer: a systematic review and meta-analysis. PLoS One, 2014; 9: e94116

21. Darwish B, Roman H. Nerve Sparing and Surgery for Deep Infiltrating Endometriosis: Pessimism of the Intellect or Optimism of the Will. Semin Reprod Med. 2017;35:72-80.

22. Lemos N, D'Amico N, Marques R, Kamergorodsky G, Schor E, Girao MJ. Recognition and treatment of endometriosis involving the sacral nerve roots. Int Urogynecol J. 2016;27:147-50.

\section{Hosted file}

Table 1 .doc available at https://authorea.com/users/316235/articles/471221-postoperativeoutcomes-following-surgery-for-deep-endometriosis-of-the-sacral-plexus-and-sciaticnerve-a-52-patient-consecutive-series

\section{Hosted file}

Table 2 .doc available at https://authorea.com/users/316235/articles/471221-postoperativeoutcomes-following-surgery-for-deep-endometriosis-of-the-sacral-plexus-and-sciaticnerve-a-52-patient-consecutive-series

\section{Hosted file}

Table 3.doc available at https://authorea.com/users/316235/articles/471221-postoperativeoutcomes-following-surgery-for-deep-endometriosis-of-the-sacral-plexus-and-sciaticnerve-a-52-patient-consecutive-series 


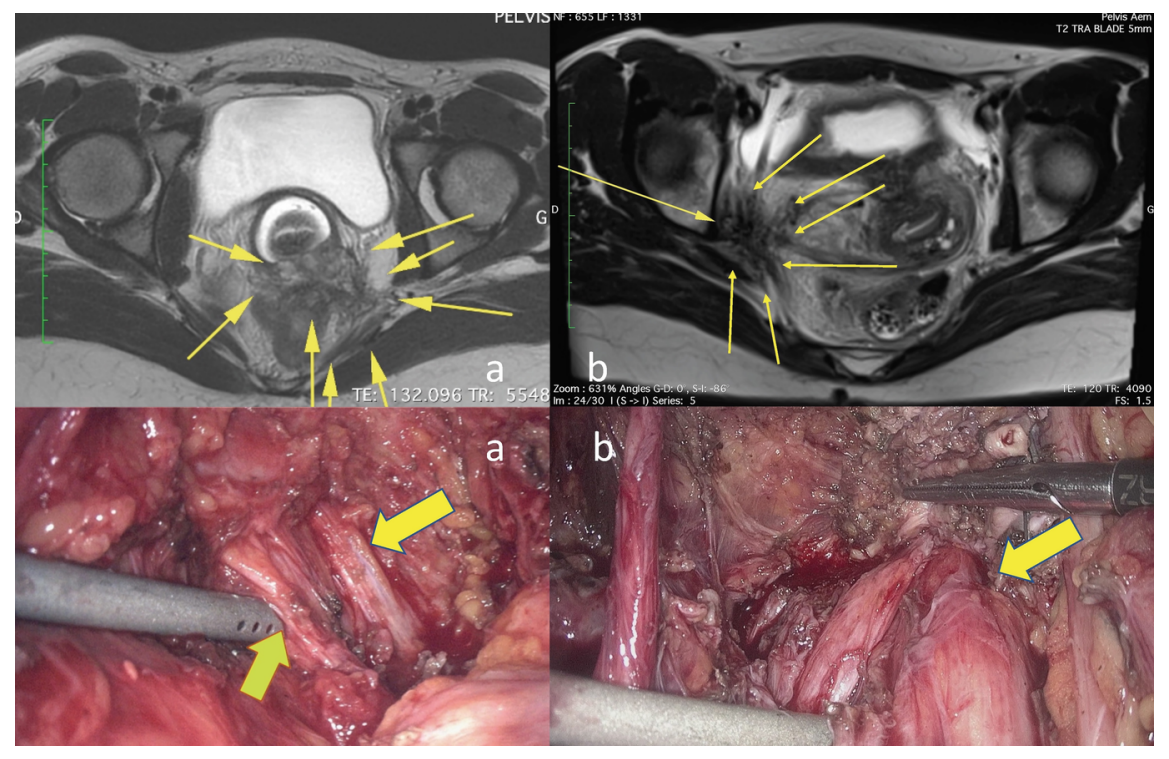

